\title{
Investigation of the natural supplement IQP-AS-119 for the prevention of upper respiratory tract infections in marathon race participants: A randomized, double-blind, placebo-controlled pilot study
}

\author{
UDO BONGARTZ ${ }^{1}$, UWE HOCHMANN ${ }^{1}$, UTE POHL $^{1}$, GORDANA BOTHE ${ }^{1}$, PATRICIA DE COSTA ${ }^{2}$, \\ PEE-WIN CHONG ${ }^{2}$, LYNETTE CHEW $^{2}$ and RALF UEBELHACK ${ }^{1}$
}

${ }^{1}$ Analyze \& Realize GmbH, D-13467 Berlin, Germany; ${ }^{2}$ InQpharm Group Sdn Bhd, Kuala Lumpur 50480, Malaysia

Received January 23, 2020; Accepted July 28, 2020

DOI: $10.3892 / \mathrm{etm} .2020 .9575$

\begin{abstract}
Intense and prolonged exercise leads to immune suppression, causing upper respiratory tract infections (URTI). A proprietary standardized dietary supplement, IQP-AS-119 has been previously developed to aid immune responses under such conditions. The current randomized, double-blind, placebo-controlled pilot study aimed to investigate the effects of IQP-AS-119 on marathon runners. A total of 80 participants were randomized equally into groups receiving either placebo (P group) or IQP-AS-119 (V group) treatment, starting 3 weeks before and for 14 days after the marathon. Benefit assessment was performed using different questionnaires. Post-marathon, the $\mathrm{V}$ and $\mathrm{P}$ groups reported $1 \pm 2.38$ and $2.11 \pm 3.25$ days with upper respiratory tract symptoms (URTS), respectively ( $\mathrm{P}=0.038)$. During the 14 days post-marathon, $20.0 \%$ of the participants in the $\mathrm{V}$ group compared with $44.4 \%$ in the $\mathrm{P}$ group reported URTS ( $\mathrm{P}=0.042)$. The V group reported significantly milder URTS compared with the P group on Days $9,12,13$ and 14 post-marathon $(\mathrm{P}<0.05)$. The total Perceived Stress Questionnaire-20 score on days 2-14 were significantly lower for the $\mathrm{V}$ group compared with the $\mathrm{P}$ group $(\mathrm{P}=0.035)$. In the Short Form 12 Health Survey, the V group exhibited significant improvement in mental composite score on days -5 to 14 compared with the $\mathrm{P}$ group $(\mathrm{P}=0.038)$. In the overall treatment
\end{abstract}

Correspondence to: Ms. Lynette Chew, InQpharm Group Sdn Bhd, Plaza Mont Kiara, No. 2, Jalan Kiara, Kuala Lumpur 50480, Malaysia E-mail: lynette@inqpharm.com

Abbreviations: FAS, full analysis set; IP, investigational products; OTE, overall treatment effect; P group, placebo group; PSQ20, Perceived Stress Questionnaire; SF-12, Short Form 12 Health Survey; QoL, quality of life; URTI, upper respiratory tract infection; URTS, upper respiratory tract symptoms; V group, IQP-AS-119 group; VCAS, valid case analysis set; WURSS-21, Wisconsin Upper Respiratory Symptom Survey 21

Key words: garlic, immune, IQP-AS-119, marathon, upper respiratory tract infection effect assessment, there were no statistically significant differences between the groups. The IQP-AS-119 was rated 'very good' or 'good' by investigators and participants, respectively, for 71 and $65 \%$ of the participants. The tolerability of IQP-AS-119 was rated as 'very good' or 'good' by both investigators and $95 \%$ of participants. No clinically relevant differences were observed between groups regarding adverse events or other safety parameters. Therefore, IQP-AS-119 was demonstrated to reduce the incidence and severity of URTI in marathon runners. Given its good tolerability profile, IQP-AS-119 may be a good nutritional supplement for the reduction of URTS in susceptible individuals.

\section{Introduction}

In defence against infections, the immune system regulates the functions of a multitude of cells and molecules to fight invading pathogens. Physical and psychological stress, sleep deprivation and malnutrition are likely to cause suboptimal immune system function, thereby creating an opportunity for infectious agents to invade the body (1-4). The effects of intense and prolonged exercise has been documented to adversely affect the immune system (5), resulting in immunosuppression lasting for 3-72 h (6), or occasionally 1-2 weeks for athletes in competition (7). The concept of an 'open window', where an individual may be more susceptible to infectious agents (8), is often used to explain the reason behind upper respiratory tract infections or symptoms (URTI or URTS) following a taxing bout of exercise (9-11). Accordingly, the use of exercise as a model for immune stress has been employed in previous studies $(12,13)$. The incidence of URTS has been reported to be higher among athletes subjected to high-intensity and endurance exercise, such as marathon and ultramarathon running, particularly in the days leading up to and after the competition $(9,14,15)$. Marathon running has been demonstrated to transiently suppress various aspects of immunity (16), and an increase in the levels of certain immune markers after a marathon race have been linked to increased susceptibility to infections (17). In particular, the URTI incidence was reported to be 2.5 - to $\sim 6$-fold higher among marathon runners in previous studies $(18,19)$. Therefore, marathon running has been employed by different research groups as a stressor to 
the immune system in order to investigate the impact of dietary supplements on the incidence and severity of URTS (20-22).

The aim of the present study was to investigate the potential health benefits of a dietary supplement, IQP-AS-119, in reducing the incidence and severity of URTS in marathon runners, providing an indication of the supplement's efficacy in aiding the body's immune system under conditions of stress, as modelled by the marathon run.

IQP-AS-119 is a dietary supplement containing a proprietary blend of ingredients, including a patent-pending standardized garlic extract, with green chireta (Andrographis paniculata), plant superoxide dismutase extract, vitamin B complex, cholecalciferol and zinc. Allicin, which is an organosulphur compound found in garlic, has been indicated to reduce the incidence of common cold symptoms in a previous 12 week study (23). Green chireta has also been demonstrated to be beneficial in improving URTI symptom scores (sore throat, sleep disturbances, cough, headache and earache) in randomized controlled trials in patients with uncomplicated URTI $(24,25)$.

Plant superoxide dismutase extracts have been demonstrated to alleviate stress $(26,27)$. Daily intake of concentrates containing superoxide dismutase has also been revealed to reduce physical and mental fatigue along with stress in healthy individuals compared with those receiving placebo treatment (26).

Cholecalciferol at $300 \mathrm{IU}$ has been indicated to reduce the risk of reported acute URTI by $50 \%$ compared with those receiving placebo treatment (28). A review on cholecalciferol supplementation suggested a daily intake of 1,000 IU per adult as a prophylaxis for respiratory tract illnesses (29).

Zinc acetate administered in the form of lozenges at the onset of a common cold has been indicated to reduce the severity and duration of cold symptoms $(30,31)$. Thiamine supplementation has also been indicated to be beneficial in reducing fatigue associated with exercise $(32,33)$. Folic acid supplementation, as demonstrated in a 12-week study, resulted in an increase in the serum levels of proteins involved in the regulation and activation of immune function (34). Based on the reports of beneficial effects of these ingredients, it was hypothesized that IQP-AS-119 may be used to support immune function and improve energy levels when the body is under stress, helping individuals maintain optimal health.

\section{Materials and methods}

Ethics and consent. The present trial was performed in accordance with the principles of the Declaration of Helsinki (35) and the EU recommendations for Good Clinical Practice (EMA/CHMP/ICH/135/95), E6 (36), and was approved by the Ethics Committee of the Charité-Universitätsmedizin Berlin. All participants provided written informed consent prior to their participation in the trial. This trial was registered at Clinicaltrials.gov as NCT02873910 (registered 22 August 2016; retrospectively registered, https://clinicaltrials. gov/ct2/show/NCT02873910).

Design. The current study was a single-centre, double-blind, randomized, placebo-controlled pilot trial involving participants in the Berlin Marathon 2016. The study began 70 days before the marathon and lasted until 25 days after the marathon. The treatment period started 3 weeks prior to and continued for
2 weeks after the Berlin Marathon on 25 September 2016. The study involved 2 visits to the study centre at the beginning and the end of the study, with 2 reminder phone calls between the visits.

Participants. Participation in the study was open to registered runners of the 2016 Berlin marathon. The aim was to recruit 80 participants to be randomized into this exploratory pilot study. No formal hypothesis testing had been prespecified. The main eligibility criteria for participation included Caucasians of either sex, aged 18-69 years, who were residing in either Berlin or Brandenburg. The participants should have successfully completed at least 1 marathon in the last 2 years, with a completion time of 3-5.5 h. The participants were required to be non-smokers, or to have ceased smoking for at least 12 months prior to the screening visit. They should also have regular sleeping patterns without suspected sleeping disorders for the 3 months prior to the screening visit. Participants with a history of severe cardiovascular disease or collapse during a running event, or during training, were excluded. Participants with any abnormalities observed in the screening exercise electrocardiogram that pointed to an increased cardiovascular risk, systolic blood pressure $\geq 140 \mathrm{mmHg}$ and/or diastolic blood pressure $\geq 90 \mathrm{mmHg}$, were considered ineligible for the pilot study. Runners with URTS, injuries and/or were unable to complete all questionnaires were excluded from the trial. Finally, a total of 80 healthy asymptomatic adults that fit the inclusion criteria participated in the pilot trial. Participants were recruited between July and August 2016.

Treatment groups. Numbered days with a minus sign were used to indicate the time period before the marathon, whereas numbered days without a minus sign were used to indicate the trial period after the marathon. Between day -70 and day -26 , the participants were randomly allocated to either the IQP-AS-119 group (V group) or placebo group (P group). The investigational product (IP) was either the IQP-AS-119 in tablet form, or a placebo tablet identical to the IQP-AS-119, which contained microcrystalline cellulose instead of the active ingredients. Participants in the V group took one tablet of IQP-AS-119 and participants in the $\mathrm{P}$ group took one placebo tablet daily from day -21 to day 14 .

During the study, the participants refrained from taking nutritional supplements, or other 'immune support' products, systemic analgesics (other than paracetamol up to 2,000 mg/day or ibuprofen up to $800 \mathrm{mg} / \mathrm{day}$ ) and antibiotics. Additionally, subjects were asked to refrain from strenuous exercise (other than completing training runs).

Assessments. The benefits of the IP were assessed by a number of questionnaires distributed during the study. An overview of the schedule of events for the trial is presented in Fig. 1.

Participants were asked 'Do you experience any health problems today?' and 'Do you think you have a cold/URTS?' in the participant diary from Day -1 to Day 14 (for example, at 14 days after the marathon). On any day that the participant answered 'yes' to having health problems, they were asked to report any ailments they were suffering from, such as gastrointestinal symptoms, muscle/joint/bone problems, injuries, allergies or other infections. 


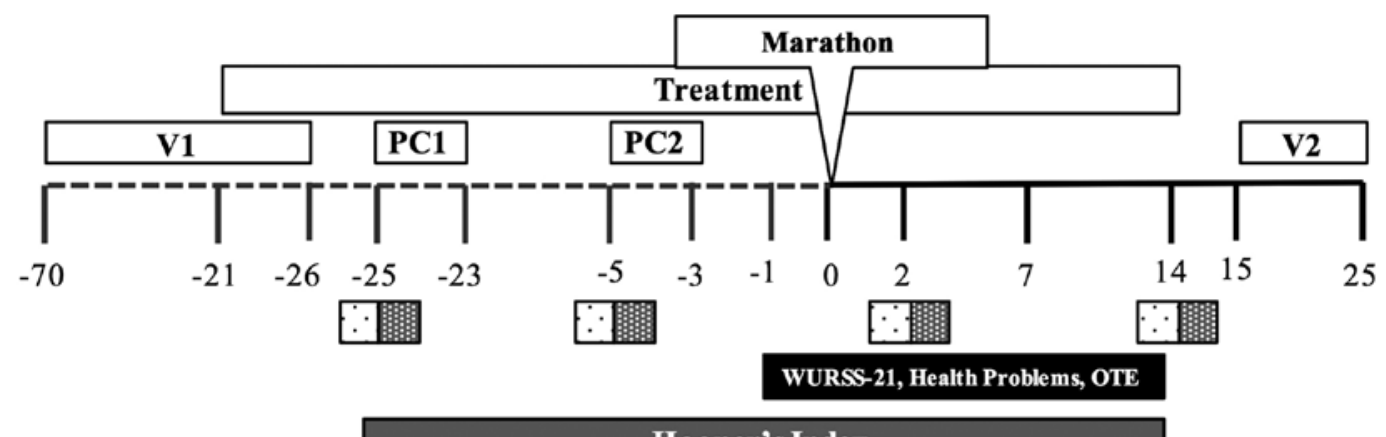

Hooper's Index

$\begin{aligned} & \text { V1 } \begin{array}{l}\text { Visit 1, including screening \& } \\ \text { randomization, Days }-70 \text { to } 26\end{array} \quad \text { V2 } \\ & \text { Visit 2, } \\ & \text { Days } 15 \text { to } 25\end{aligned} \quad$ PC1 $\begin{aligned} & \text { Phone call 1, } \\ & \text { Days }-25 \text { to }-23\end{aligned} \quad$ PC2 $\begin{aligned} & \text { Phone call 2, } \\ & \text { Days }-5 \text { to }-3\end{aligned}$
Daily subject diary on URTS, WURSS-21, health problems and OTE from Days -1 to 14
$\square$ Hooper's Index (fatigue, sleep, stress, muscle soreness) from Days -25 to 14
$\therefore$ PSQ20 (perceived stress) was assessed on Days $-25,-5,2$ and 14
$\square$ SF-12 (quality of life) was assessed on Days $-25,-5,2$ and 14

Figure 1. Schedule of study events. OTE, overall treatment effect; PSQ20, Perceived Stress Questionnaire; SF12, Short Form 12 Health Survey; URTS, upper respiratory tract symptoms; WURSS-21, Wisconsin Upper Respiratory Symptom Survey 21; PC, phone call; V, visit.

Any day of answering 'yes' to having a cold/URTS was considered as a URTS day. Furthermore, in the daily participant diary from Day -1 to Day 14, the Wisconsin Upper Respiratory Symptom Survey (WURSS-21) was also completed. The WURSS-21 is a validated illness-specific instrument used to assess the negative impact of acute URTI that is presumed to be viral (for example, the common cold) on the participant's quality of life (QoL) (37). Item 1 in the WURSS-21 asked the participants 'How sick do you feel today?', items 2-11 assessed the participants' severity of URTS, while items 12-20 assessed the extent to which the participants' symptoms interfered with daily activities. Each item was to be rated by on a scale of 0 (no symptom/not affected), to 7 (severe). Item 21 in the WURSS-21 asked the participants to assess whether their symptoms were better or worse compared with the day before. For each URTS day, the severity was evaluated using the WURSS-21.

At the end of the first week (day 7) and second week (day 14) after the marathon, the participants were asked to indicate whether their health had improved, remained the same, or worsened since the marathon (day 0) using the overall treatment effect (OTE) assessment (38). Participants were asked 'How has your health changed since your participation in the marathon?' and they rated their response on a 15-point global rating scale from -7 (a great deal worse), through 0 (no change) to +7 (a great deal better) (38). The change of the participants' scores was calculated by taking the difference between the scores for day 14 minus day 7 .

On each day of the trial, from day -25 through to day 25 , the participants assessed their well-being by rating their fatigue, stress, delayed onset of muscle soreness, and sleep quality/disorders on a scale of 1 to 7 for each of the 4 items in the Hooper's Index (39). The Hooper's Index is the summation of the 4 ratings.

Additionally, the participants filled out the Perceived Stress Questionnaire (PSQ20) (40) and the Short Form 12 Health Survey Questionnaire (SF-12) (41) on days -25, -5, 2 and 14 of the trial. The PSQ20 had 20 items in 4 scales: Worries, tension, joy and demands. For each item, the participants were asked to evaluate the frequency of occurrence of each item during the preceding week using values from 1 (almost never) to 4 (usually). The total score of the items for worries, tension, joy and demands was calculated from the participants' ratings and transformed according to the instructions provided by the questionnaire's developers (40), resulting in transformed scores ranging from 0 to 100 . The total PSQ20 score was used to estimate the participants' stress levels. The SF-12 assessed the participants' general well-being during the preceding week, with evaluation of 12 items clustered into 2 scores for the physical and mental composites according to the questionnaire's instructions (41). The changes to the composite scores were calculated by taking the mean composite scores on day 14 minus the mean composite scores on either day -5 or day 2 .

At the end of the study, both the participants and investigators independently evaluated the benefit of the IP by means of a global scale ('very good', 'good', 'moderate' and 'poor').

The assessment of tolerability and safety included physical examination, recording of vital signs at each visit, and analysis of full blood count parameters, liver and renal function parameters (alanine transaminase, aspartate aminotransferase, $\gamma$-GT, alkaline phosphatase, bilirubin, creatinine, urea and uric acid), carbohydrate and lipid metabolism parameters at the beginning and at the end of the study.

Statistical analysis. The results are presented as the means \pm standard deviation. Non-parametric tests were used for the analyses of the differences between groups, namely the Mann-Whitney U-test for independent groups and the $\chi^{2}$ test for the comparison of percentages. The Kaplan-Meier method was used to compare differences between groups for cumulative URTS days and Hooper's indices. Both the full analysis set (FAS) and valid case analysis set (VCAS) were analysed 
Assessed for eligibility (82)

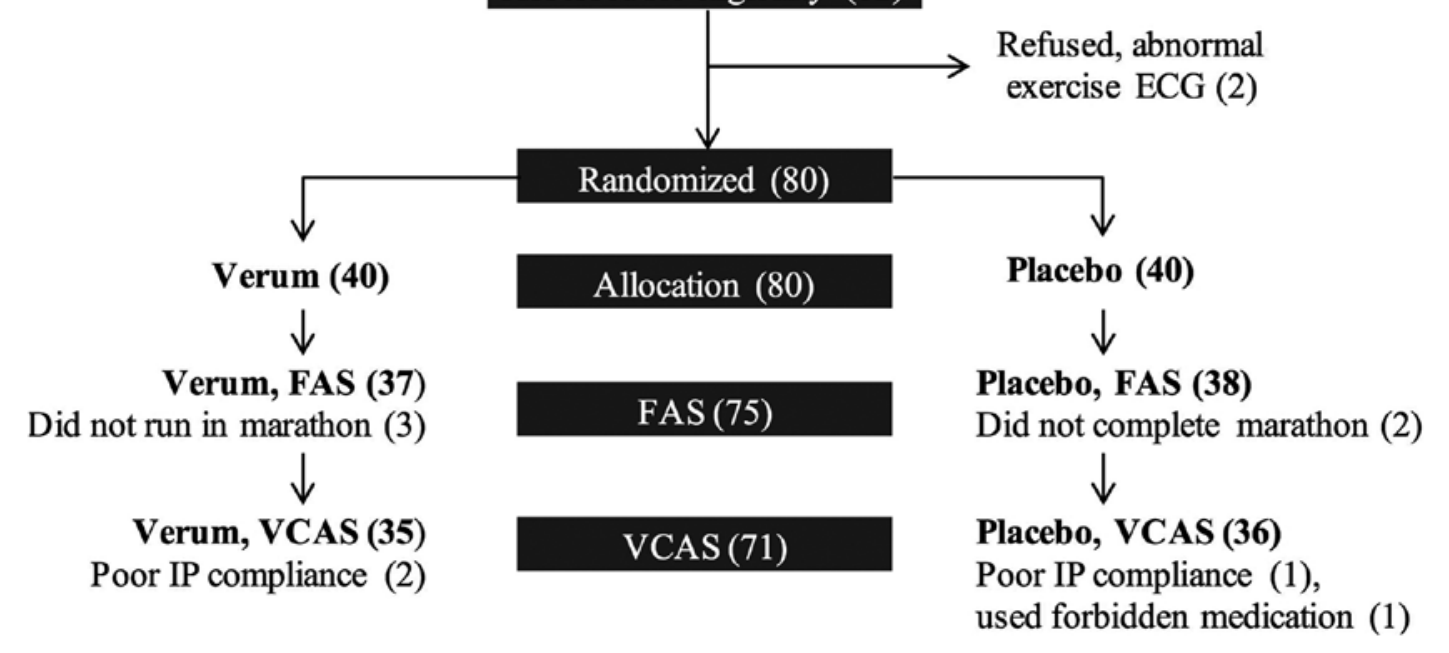

Figure 2. Disposition of participants. Number of participants is provided in parenthesis. ECG, electrocardiogram; FAS, full analysis set; IP, investigational products; VCAS, valid case analysis set.

Table I. Participant characteristics.

\begin{tabular}{lcc}
\hline Characteristics & V group $(\mathrm{n}=37)$ & P group $(\mathrm{n}=38)$ \\
\hline Sex & & \\
Men & $21(56.8 \%)$ & $22(57.9 \%)$ \\
Women & $16(43.2 \%)$ & $16(42.1 \%)$ \\
Age (years) & $43.3 \pm 10.2$ & $40.4 \pm 10.3$ \\
Height $(\mathrm{cm})$ & $175.4 \pm 11.1$ & $173.6 \pm 8.7$ \\
Body weight $(\mathrm{kg})$ & $72.1 \pm 11.5$ & $68.8 \pm 10.3$ \\
BMI $\left(\mathrm{kg} / \mathrm{m}^{2}\right)$ & $23.29 \pm 1.94$ & $22.72 \pm 2.04$ \\
\hline
\end{tabular}

FAS population $(n=75)$. Values are reported as means \pm standard deviation. No significant differences were observed between groups $(\mathrm{P}>0.05)$ for any of the characteristics. FAS, full analysis set.

in order to detect any differences between them (42). In cases where there was a major non-compliance of the protocol, the subject data was excluded from the FAS and only included in the VCAS analyses. All statistical analyses were performed using SPSS, version 22.0 (IBM Corp.). All tests were performed with a significance level (type I error) of $5.0 \%$ (two-tailed test).

\section{Results}

Participants. A total of 82 individuals were assessed for eligibility, and 80 finally participated in the present pilot study. The participants who completed the marathon were 43 men and 32 women, aged $22-64$ years (mean age, $42.4 \pm 10.2$ years). Three participants from the $\mathrm{V}$ group did not run in the marathon and 2 participants in the $\mathrm{P}$ group did not complete the marathon, and they were excluded from the full FAS. By the end of the study, 2 participants from each group either did not comply with the intake of IP or had used excluded medication, and they were excluded from the VCAS. The participant disposition flow chart is presented in Fig. 2.
Participants from the V and $\mathrm{P}$ groups did not differ significantly in terms of mean age, height, body weight and body mass index (Table I).

URTS. The incidence of URTS reported by participants in the $\mathrm{V}$ group was lower compared with that in the $\mathrm{P}$ group (Table II). In the FAS population, the percentage of participants who had at least 1 day of URTS between day 1 and day 14 following the marathon was lower by $23.1 \%$ in the $\mathrm{V}$ group compared with the $\mathrm{P}$ group $(\mathrm{P}=0.05)$. Additionally, participants in the $\mathrm{P}$ group reported $48.3 \%$ more days with URTS between day 1 and day 14 post-marathon $(\mathrm{P}=0.052)$. In the VCAS population, the percentage of reported URTS and the number of days of URTS differed significantly between the two groups $(\mathrm{P}<0.05)$, with the $\mathrm{V}$ group reporting more favourable results (Table II). The P group had $2.11 \pm 3.25$ days with URTS, twice more when compared with the $\mathrm{V}$ group with $1.00 \pm 2.38$ days $(\mathrm{P}=0.038)$.

WURSS-21. Changes to the score for URTS, listed as items 2-11 in the WURSS-21, were evaluated by calculating the mean scores on the day of assessment minus the score on day -1 . Days with milder URTS exhibited lower scores and the negative values, representing an improvement of URTS on the day of assessment compared with day -1. URTS that were assessed by the questionnaire revealed that participants who had taken IQP-AS-119 reported significantly milder URTS on days 9, 12, 13 and 14 when compared to participants in the $\mathrm{P}$ group $(\mathrm{P}<0.05)$ (Table III). Changes from baseline (day -1$)$ to day 1 through day 8 were not statistically significant (data not shown).

Hooper's index. The summation of the four items (sleep, muscle soreness, stress, fatigue) was used to yield the Hooper's Index from the responses of 72 participants on day 7 and 74 participants on day 14, out of the 75 participants in the FAS. The mean Hooper's Index at the end of the first and second weeks after the marathon exhibited no statistically significant differences between the two groups (Table IV). 
Table II. Participants with at least 1 day of URTS reported between Day 1 and Day 14 post-marathon.

\begin{tabular}{|c|c|c|c|c|c|c|}
\hline \multirow[b]{2}{*}{ Factor } & \multicolumn{3}{|c|}{ FAS Population } & \multicolumn{3}{|c|}{ VCAS Population } \\
\hline & $V$ group $(n=37)$ & $P$ group $(n=38)$ & P-value & $V$ group $(n=35)$ & $P$ group $(n=36)$ & P-value \\
\hline \multicolumn{7}{|l|}{ Proportion of participants } \\
\hline No URTS & $78.4 \%$ & $55.3 \%$ & 0.050 & $80.0 \%$ & $55.6 \%$ & 0.042 \\
\hline$\geq 1$ day URTS & $21.6 \%$ & $44.7 \%$ & & $20.0 \%$ & $44.4 \%$ & \\
\hline Number of days with URTS/cold ${ }^{1}$ & $1.05 \pm 2.37$ & $2.03 \pm 3.18$ & 0.052 & $1.00 \pm 2.38$ & $2.11 \pm 3.25$ & 0.038 \\
\hline
\end{tabular}

The number of days is reported as means \pm standard deviation. FAS, full analysis set; URTS, upper respiratory tract symptoms; VCAS, valid case analysis set.

Table III. Changes in WURSS-21, mean score (items 2-11) from baseline (Day -1).

\begin{tabular}{lccc}
\hline Day & V group $(\mathrm{n}=37)$ & P group $(\mathrm{n}=38)$ & P-value \\
\hline $\begin{array}{l}\text { Day 0 } \\
\text { (marathon day) }\end{array}$ & $-0.15 \pm 0.42$ & $-0.04 \pm 0.15$ & 0.400 \\
Day 9 & $-0.15 \pm 0.63$ & $0.14 \pm 0.91$ & 0.026 \\
Day 10 & $-0.14 \pm 0.68$ & $0.10 \pm 0.87$ & 0.158 \\
Day 11 & $-0.20 \pm 0.59$ & $0.12 \pm 0.83$ & 0.051 \\
Day 12 & $-0.19 \pm 0.61$ & $0.11 \pm 0.88$ & 0.031 \\
Day 13 & $-0.16 \pm 0.70$ & $0.07 \pm 0.82$ & 0.019 \\
Day 14 & $-0.17 \pm 0.67$ & $0.09 \pm 0.88$ & 0.028 \\
\hline
\end{tabular}

Values are reported as means \pm standard deviation. Only changes on Day 0, and Days 9-14 are shown. Changes of scores were calculated by taking the mean scores of items 2-11 on the day of assessment minus mean scores on Day -1. Days with milder upper respiratory tract symptoms would have lower scores, the negative values thus represent an improvement of upper respiratory symptoms on the day of assessment compared to Day -1. WURSS-21, Wisconsin Upper Respiratory Symptom Survey 21

$P S Q 20$. The scales that had higher values indicated greater feelings of 'joy', 'worries', 'tensions' and 'demands'. Changes to the scores for the individual scales and the total score were calculated by taking the mean scores on day 14 minus the mean scores on either day -5 or day 2 , so a lower score on day 14 would result in negative numbers. Increased scores of 'joy', and decreased scores of 'worries', 'tension', 'demand' and 'total score' indicated improvement to participants' QoL. The reduction of scores for the scales 'tension' and 'demands' were not significantly different between the $\mathrm{V}$ and $\mathrm{P}$ groups. In the assessment of 'joy' in the VCAS population, improvement in ratings was reported in the $\mathrm{V}$ group, while deterioration was reported in the $\mathrm{P}$ group from day 2 to day 14 , and the difference was statistically significant $(\mathrm{P}=0.021)$. The similar assessment in the FAS population did not reveal statistically significant differences (data not shown). The reduction in the scale for 'worries' was statistically increased in the $\mathrm{V}$ group compared with the $\mathrm{P}$ group from day -5 to day 14 and from day 2 to day $14(\mathrm{P}=0.021$ and $\mathrm{P}=0.012$, respectively) in the VCAS
Table IV. Summation of mean scores for Hooper's Index in the FAS population.

\begin{tabular}{lccc}
\hline $\begin{array}{l}\text { Day post } \\
\text { marathon }\end{array}$ & V group & P group & P-value \\
\hline Day 7 & $9.64^{\mathrm{a}} \pm 3.55$ & $8.61^{\mathrm{a}} \pm 2.91$ & 0.402 \\
Day 14 & $9.57^{\mathrm{b}} \pm 3.54$ & $9.19^{\mathrm{b}} \pm 3.40$ & 0.675 \\
\hline
\end{tabular}

${ }^{\mathrm{a}} 36$ participants; ${ }^{\mathrm{b}} 37$ participants. Values are reported as means \pm standard deviation. FAS, full analysis set.

population (Table V). The analysis in the FAS population for the scores of 'worries' revealed similar statistically significant findings $(\mathrm{P}<0.05)($ data not shown). As regards to the total PSQ20 score, the $\mathrm{V}$ group reported an improvement from day 2 to day 14 compared with the $\mathrm{P}$ group $(\mathrm{P}=0.035)$ in the VCAS population.

SF-12, OTE and assessment of benefit and tolerability. The items in the SF-12 questionnaire were analysed by the physical composite (items 1, 2, 3, 4, 5 and 8), and mental composite scores (items 6, 7, 9, 10,11 and 12). The changes to the composite scores were calculated by taking the mean composite scores on day 14 minus the mean composite scores on either day -5 or day 2 . Neither group had significant differences regarding changes to the physical composite score. For the mental composite, the $\mathrm{V}$ group exhibited a significant improvement from day -5 to day 14 compared with the $\mathrm{P}$ group $(\mathrm{P}=0.038$; Table VI).

For each week after the marathon, the OTE scores did not differ significantly between groups (all $\mathrm{P}>0.05$, Table VII). A negative value in the score for day 7 indicated rating of worsening health since the marathon. The change of the participants' scores between the 2 days of assessment was calculated by taking the difference between the scores for day 14 minus day 7 .

In the global assessment of benefit, IQP-AS-119 was considered as 'very good' or 'good' for 71 and $65 \%$ of the participants, as rated by the investigators and participants, respectively (Fig. 3). The respective ratings for the placebo were $45 \%$ (rating by investigators) and $42 \%$ (rating by participants) (data not shown). The ratings did not differ significantly between investigators and participants $(\mathrm{P}>0.05)$. 
Table V. Changes to PSQ20 for joy, worries, tension and demands; and total score in the VCAS population.

\begin{tabular}{|c|c|c|c|}
\hline Changes to PSQ20 & V group & $\mathrm{P}$ group & P-value \\
\hline \multicolumn{4}{|l|}{ Joy } \\
\hline Day -5 to 14 & $3.92^{\mathrm{a}} \pm 13.84$ & $-0.95^{\mathrm{b}} \pm 13.25$ & 0.160 \\
\hline Day 2 to 14 & $1.52 \pm 9.71$ & $-2.96 \pm 9.35$ & 0.021 \\
\hline \multicolumn{4}{|l|}{ Worries } \\
\hline Day -5 to 14 & $-4.51^{\mathrm{a}} \pm 10.73$ & $1.14^{\mathrm{b}} \pm 9.63$ & 0.021 \\
\hline Day 2 to 14 & $-2.29 \pm 9.56$ & $4.07 \pm 9.47$ & 0.012 \\
\hline \multicolumn{4}{|l|}{ Tension } \\
\hline Day -5 to 14 & $-2.35^{\mathrm{b}} \pm 11.59$ & $2.75^{\mathrm{c}} \pm 15.05$ & 0.204 \\
\hline Day 2 to 14 & $-2.67 \pm 11.11$ & $0.76 \pm 13.11$ & 0.419 \\
\hline \multicolumn{4}{|l|}{ Demands } \\
\hline Day -5 to 14 & $-3.43^{\mathrm{d}} \pm 18.79$ & $-0.76^{\mathrm{b}} \pm 15.32$ & 0.718 \\
\hline Day 2 to 14 & $-4.51 \pm 17.42$ & $2.04 \pm 14.59$ & 0.170 \\
\hline \multicolumn{4}{|l|}{ Total Score } \\
\hline Day -5 to 14 & $-3.59^{\mathrm{d}} \pm 11.34$ & $0.93^{\mathrm{a}} \pm 9.94$ & 0.110 \\
\hline Day 2 to 14 & $-2.75 \pm 9.51$ & $2.33 \pm 8.22$ & 0.035 \\
\hline
\end{tabular}

${ }^{\mathrm{a}} 34$ participants; ${ }^{\mathrm{b}} 35$ participants; 36 participants; ${ }^{\mathrm{d}} 33$ participants. Values are reported as means \pm standard deviation. For each individual scale, higher values indicated greater feelings of 'joy', 'worries', 'tensions' or 'demands'. Changes to the scores of the individual scales and the total score were calculated by taking the mean scores on Day 14 minus the mean scores on either Day -5 or Day 2. Thus, lower mean scores on Day 14 resulted in negative numbers. In the table, a positive change of 'joy', and a negative change of 'worries', 'tension', 'demand' and 'total score' indicated improvement to the participants' quality of life. PSQ20, Perceived Stress Questionnaire; VCAS, valid case analysis set.

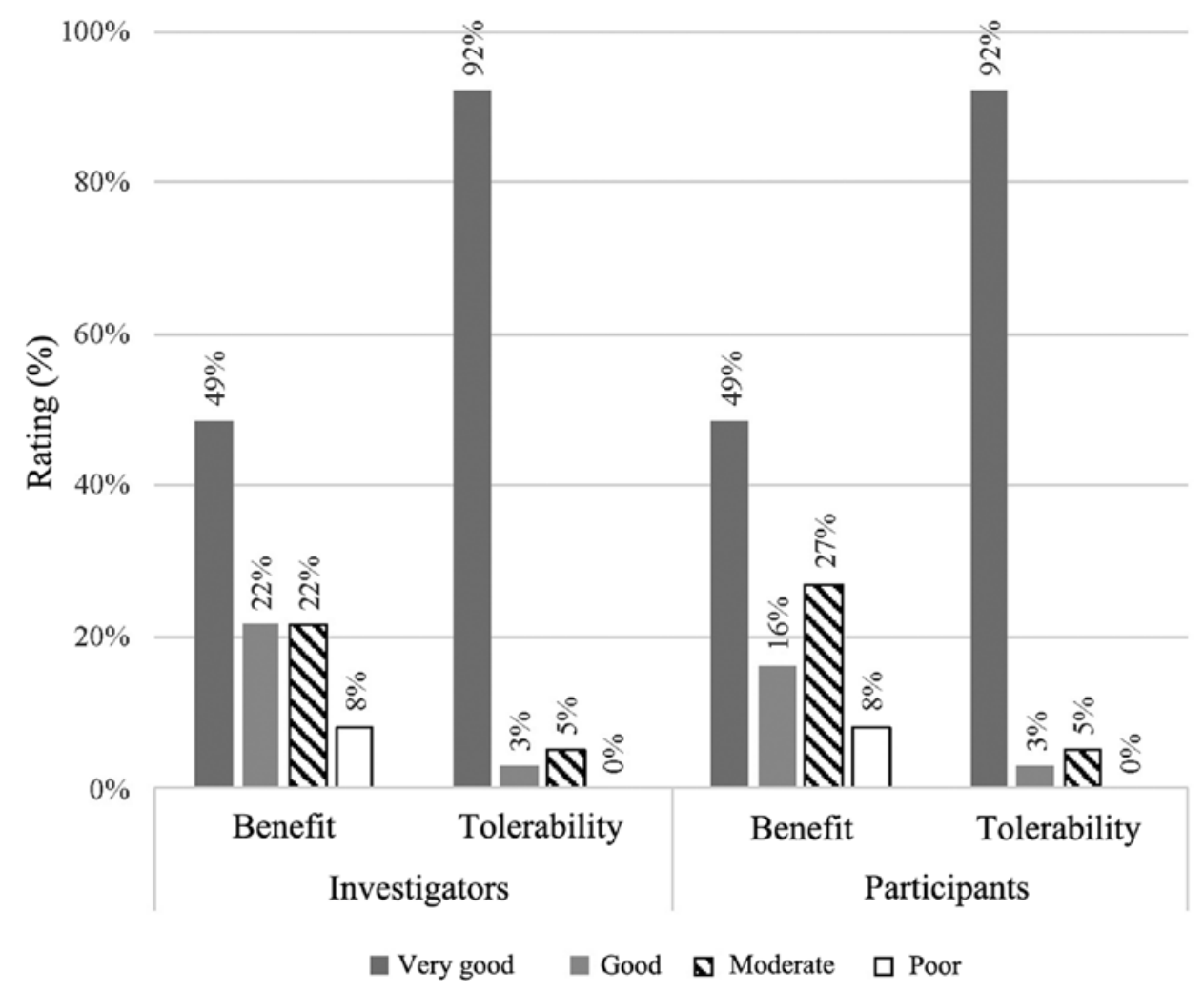

Figure 3. Global assessment of benefits and tolerability of IQP-AS-119. The differences in ratings for benefits and tolerability were not statistically significant between investigators and participants $(\mathrm{P}>0.05)$.

In the assessment of adverse events, physical examination, recording of vital signs and other laboratory parameters, there were no clinically relevant differences between the $\mathrm{V}$ and P groups. The tolerance of IQP-AS-119 was rated as 'very good' 
Table VI. Changes to physical and mental composite scores in the SF-12 in the FAS population.

A, PCS

\begin{tabular}{lccc}
\hline Changes to SF-12 & V group $(\mathrm{n}=35)$ & P group $(\mathrm{n}=35)$ & P-value \\
\hline Day -5 to 14 & $-0.60 \pm 8.96$ & $-1.53 \pm 5.68$ & 0.449 \\
Day 2 to 14 & $0.38 \pm 8.58$ & $1.36 \pm 9.19$ & 0.440 \\
\hline
\end{tabular}

\section{$\mathrm{B}, \mathrm{MCS}$}

\begin{tabular}{lccc}
\hline Changes to SF-12 & V group $(n=35)$ & P group $(n=35)$ & P-value \\
\hline Day -5 to 14 & $2.13 \pm 7.11$ & $-0.33 \pm 4.51$ & 0.038 \\
Day 2 to 14 & $-0.39 \pm 6.04$ & $-1.47 \pm 3.55$ & 0.271 \\
\hline
\end{tabular}

Values are reported as means \pm standard deviation. Changes to the composite scores were calculated by taking the composite scores on Day 14 minus the mean composite scores on either Day -5 or Day 2. Lower composite scores on Day 14 indicated a worse health status, resulting in negative values in the computed differences from Day -5 or Day 2, whereas improvement in health status was indicated by a positive difference in scores. FAS, full analysis set; SF-12, Short Form 12 Health Survey; MCS, mental composite score; PCS, physical composite score.

Table VII. Mean OTE scores for first and second week after the marathon in the FAS population.

\begin{tabular}{lccc}
\hline Day & V group $(n=36)$ & P group $(n=38)$ & P-value \\
\hline D7 & $0.33 \pm 2.19$ & $-0.11 \pm 2.13$ & 0.536 \\
D14 & $0.86 \pm 2.27$ & $0.34 \pm 2.88$ & 0.343 \\
D14-D7 & $0.53 \pm 1.86$ & $0.45 \pm 2.39$ & 0.705
\end{tabular}

Values are reported as means \pm standard deviation. The overall treatment effect was assessed with the question 'How has your health changed since your participation in the marathon?' using a 15-point global rating scale from -7 (a great deal worse) through 0 (no change) to +7 (a great deal better). Negative values in scores for Day 7 or Day 14 indicated worsening health since the marathon, whereas the Day 14 minus Day 7 score indicated changes from Day 7 to Day 14. D, day; FAS, full analysis set; OTE, overall treatment effect.

or 'good' for $95 \%$ of participants by both investigators and subjects (Fig. 3). All participants agreed with the investigators on the tolerability ratings $(\mathrm{P}=1.000)$.

\section{Discussion}

The results clearly demonstrated that runners using IQP-AS-119 had a lower incidence of URTS in the 2 weeks following the marathon. URTS were reported by $21.6 \%$ of the participants taking IQP-AS-119 compared with $44.7 \%$ of those taking the placebo in the FAS population (Table II). This was similar in the VCAS population, where the number of participants in the $\mathrm{V}$ group that exhibited URTS following the marathon was $45 \%$ of that in the P group. Additionally, the number of days with URTS reported by participants taking IQP-AS-119 was 52.6\% shorter of that experienced by the subjects in the $\mathrm{P}$ group. Previous dietary supplements had reported similar reduction of URTS severity (ranging from 33 to $49.5 \%$ as compared to placebo) and shorter duration of days with cold (ranging from 29.4 to $42.3 \%$ when compared with the placebo group) $(15,22,30)$.

In the assessment of the URTS severity per WURSS-21 (Table III), relative differences were reported in favour of IQP-AS-119 for the URTS score (items 2-11) on post-marathon days $9,12,13$ and 14 . These results suggest that a larger study population may be required to demonstrate significant differences in WURSS-21, particularly in the specific setting of post-exercise URTS, which are usually of a lesser magnitude compared with those in the context of the classical URTI. The current study used a non-invasive method for the assessment of URTI and the WURSS-21 was indicated to be appropriate and accurate. A statistically significant association was identified between the questionnaire and the laboratory-assessed biomarkers of induced rhinovirus infection in a previous study involving 399 participants (43). Moreover, the WURSS-21 is a validated (37) assessment for URTI and had been used in numerous clinical trials for assessing the treatment of the common cold (44-47).

The overall Hooper's Index assessed on the first and second week after the marathon did not reveal significant differences between the groups (Table IV). The results of the assessment of individual PSQ20 scales was indicated to be in favour of IQP-AS-119, specifically the 'joy' scale (days 2 to $14 ; \mathrm{P}=0.021$ ) and 'worries' scale (days 2 to 14; $\mathrm{P}=0.012$ ) when compared with placebo (Table V). In addition, the mental composite score of the SF-12 demonstrated the positive effect of IQP-AS-119 over placebo (Days -5 to $14 ; \mathrm{P}=0.038$; Table VI).

Collectively, the WURSS-21, total PSQ20 score and the mental composite score of the SF-12 indicated that improvements in well-being were noticeable at 2 weeks after the marathon by runners who were receiving IQP-AS-119. These observations were in line with previously observed effects of natural health products in marathon runners, such as improvement of Profile of Mood States for confusion, fatigue, tension, anger and vigour (22). 
Milder URTS experienced by participants who consumed IQP-AS-119 on days 9, 12, 13 and 14, together with reduced number of days with URTS, were consistent with other reported results of studies on garlic (23) and green chireta $(25,48)$.

Previous studies by Carillon et al (26) and Milesi et al (27) in healthy subjects demonstrated the ability of plant superoxide dismutase extract to significantly reduce stress and fatigue over placebo treatment. The PSQ20's subscores for 'joy' and 'worries' were consistent with the findings of Carillon et al and Milesi et al, where the $\mathrm{V}$ group reported significantly better 'joy' between day 2 and day 14, and lower 'worries' from day -5 to day 14 and from day 2 to day 14, compared with the placebo group.

To the best of our knowledge, the present study was the first to demonstrate that supplementation with IQP-AS-119 in immunosuppressed post-marathon runners was beneficial in reducing the incidence of URTS. A study by Cox et al (49) demonstrated that illness-prone athletes exhibited higher expression of the pro-inflammatory cytokine IL-6. These athletes reported at least 4 occurrences of URTS annually. This population is likely to benefit from the consumption of IQP-AS-119 to modulate immune response (50).

The aim of the present pilot study was to provide initial data on the beneficial effects of the IQP-AS-119 on immune health and its main limitation was the small sample size. The participants, who were marathon runners, tended to be in a good physical state and this may not accurately represent the general population. However, marathon running was used as a model of immune stress to induce URTS, and the results of the present study may be applicable to non-athletes who are susceptible to URTS. Future research should be conducted in the wider population focusing on the benefits of IQP-AS-119 in alleviating URTS. Of note, it was hypothesized that the participants maintained a regular diet, but this was not assessed. The multifactorial nature of immune regulation was indicated to be affected by stress, nutrition and sleep patterns, which would require further consideration in future studies (51). Subject-reported outcomes, such as those used in the present study, are often employed in trials evaluating supplements for the common cold $(20,22,24,27)$. The inclusion of immune markers and inflammatory markers could provide objective insights for future similar trials $(20,21,52,53)$. Furthermore, a recent review had also called for multi-omics studies for a more comprehensive understanding of the biological pathways underlying immune regulation (44).

In conclusion, IQP-AS-119 was investigated in terms of its health benefits in reducing the incidence and severity of URTS in marathon runners, providing encouraging evidence supporting the beneficial role of IQP-AS-119 in the immune response. In addition, given the good tolerability profile of IQP-AS-119 over a period of 5 weeks, it may be considered as an attractive nutritional option for prevention or reduction of URTS in susceptible individuals.

\section{Acknowledgements}

The authors would like to thank Dr Norman Bitterlich (Medizin \& Service GmbH), Dr Yvette Röske (analyze and realize $\mathrm{GmbH}$ ) and $\mathrm{Mr}$. Constantin Erlenbeck (analyze and realize $\mathrm{GmbH}$ ) for their assistance in this study.

\section{Funding}

InQpharm Group provided funding for the following: Design and conduct of the study, collection and analysis of data and writing of the manuscript.

\section{Availability of data and materials}

The datasets used and/or analyzed during the current study are available from the corresponding author on reasonable request.

\section{Author's contributions}

RU, UB and UH were responsible for data collection and management. UP, PWC and PDC contributed to study conception and design. GB managed the data analysis. LC interpreted the data and wrote the manuscript. GB, PWC and PDC critically revised and contributed to the final content of the manuscript. All authors have read and approved the final manuscript.

\section{Ethics approval and consent to participate}

Prior to start of the current study, ethical approval was gained from 'Ethikkomission der Charité' of the Charité-Universitätsmedizin Berlin, Germany. All participants gave their informed consent prior to their participation in the trial. This trial was registered at clinicaltrials.gov as NCT02873910.

\section{Patient consent for publication}

Not applicable.

\section{Competing interests}

CPW, PDC and LC are employees of InQpharm Group. The other authors declare that they have no competing interests.

\section{References}

1. Bhaskaram P: Micronutrient malnutrition, infection, and immunity: An overview. Nutr Rev 60: S40-S45, 2002.

2. Segerstrom SC and Miller GE: Psychological stress and the human immune system: A meta-analytic study of 30 years of inquiry. Psychol Bull 130: 601-630, 2004.

3. Diment BC, Fortes MB, Edwards JP, Hanstock HG, Ward MD, Dunstall HM, Friedmann PS and Walsh NP: Exercise intensity and duration effects on in vivo immunity. Med Sci Sport Exerc 47: 1390-1398, 2014.

4. Besedovsky L, Lange T and Haack M: The sleep-immune crosstalk in health and disease. Physiol Rev 99: 1325-1380, 2019.

5. Milne KJ: The immune system and its response to acute and chronic exercise. University of Windsor, 2018.

6. Kakanis M, Peake J, Hooper S, Gray B and Marshall-Gradisnik S: The open window of susceptibility to infection after acute exercise in healthy young male elite athletes. In: 2010 Asics Conference of Science and Medicine in Sport, 13: ppe84-e85, 2010.

7. Boffi El and Amari E: Upper respiratory tract infections and sports. Rev Med Suisse 6: 1499-1503, 2010 (In French).

8. Nieman DC: Physical activity, fitness and infection. In: Pysical Activity, Fitness, and Health. Human Kinetics Publishers, Champaign, IL, pp796-813, 1994.

9. Peters EM and Bateman ED: Ultramarathon running and upper respiratory tract infections. An epidemiological survey. South African Med J 64: 582-584, 1983.

10. Kuipers $\mathrm{H}$ and Keizer HA: Overtraining in elite athletes: Review and directions for the future. Sport Med 6: 79-92, 1988. 
11. Meeusen R, Duclos M, Foster C, Fry A, Gleeson M, Nieman D, Raglin J, Rietjens G, Steinacker J, Urhausen A, et al: Prevention, diagnosis, and treatment of the overtraining syndrome: Joint consensus statement of the European College of Sport Science and the American College of Sports Medicine. Med Sci Sports Exerc 45: 186-205, 2013.

12. Hoffman-Goetz L and Pedersen BK: Exercise and the immune system: A model of the stress response? Immunol Today 15: 382-387, 1994.

13. Pedersen BK and Hoffman-Goetz L: Exercise and the immune system: Regulation, integration, and adaptation. Physiol Rev 80: 1055-1081, 2000

14. Nieman DC: Exercise, upper respiratory tract infection, and the immune system. Med Sci Sport Exerc 26: 128-139, 1994.

15. Peters E, Joseph L, Peters E, Goetzsche J and Noakes T: Vitamin $\mathrm{C}$ as effective as combinations of anti-oxidant nutrients in reducing symptoms of upper respiratory tract infection in ultramarathon runners. J Sports Med 3: 23-27, 1996.

16. Rehm KE, Elci OU, Hahn K and Marshall GD: The impact of self-reported psychological stress levels on changes to peripheral blood immune biomarkers in recreational marathon runners during training and recovery. Neuroimmunomodulation 20: 164-176, 2013.

17. Gonçalves CA, Dantas PM, dos Santos IK, Dantas M, da Silva DC, Cabral BG, Guerra RO and Júnior GB: Effect of acute and chronic aerobic exercise on immunological markers: A systematic review. Front Physiol 10: 1602, 2020.

18. Nieman DC, Johanssen LM, Lee JW and Arabatzis K: Infectious episodes in runers before and after the Los Angeles Marathon. J Sports Med Phys Fitness 30: 316-328, 1990.

19. Robson-Ansley P, Howatson G, Tallent J, Mitcheson K, Walshe I, Toms C, Du Toit G, Smith M and Ansley L: Prevalence of allergy and upper respiratory tract symptoms in runners of the London Marathon. Med Sci Sports Exerc 44: 999-1004, 2012.

20. McFarlin BK, Carpenter KC, Davidson T and McFarlin MA: Baker's yeast beta glucan supplementation increases salivary $\operatorname{Ig} \mathrm{A}$ and decreases cold/flu symptomatic days after intense exercise. J Diet Suppl 10: 171-183, 2013.

21. Scherr J, Nieman DC, Schuster T, Braun S, Wolfarth B and Halle M: Non-alcoholic beer reduces inflammation and the incidence of upper respiratory tract infections after a marathon. Med Sci Sports Exerc 44: 18-26, 2012.

22. Talbott $S$ and Talbott J: Effect of BETA 1, 3/1, 6 GLUCAN on upper respiratory tract infection symptoms and mood state in marathon athletes. J Sport Sci Med 8: 509-515, 2009.

23. Josling P: Preventing the common cold with a garlic supplement: A double-blind, placebo controlled survey. Adv Ther 18: 189-193, 2001

24. Saxena RC, Singh R, Kumar P, Yadav SC, Negi MP, Saxena VS Joshua AJ, Vijayabalaji V, Goudar KS, Venkateshwarlu K and Amit A: A randomized double blind placebo controlled clinical evaluation of extract of Andrographis paniculata (KalmCold) in patients with uncomplicated upper respiratory tract infection. Phytomedicine 17: 178-185, 2010.

25. Melchior J, Palm S and Wikman G: Controlled clinical study of standardized Andrographis paniculata extract in common cold-a pilot trial. Phytomedicine 3: 315-318, 1997.

26. Carillon J, Notin C, Schmitt K, Simoneau G and Lacan D: Dietary supplementation with a superoxide dismutase-melon concentrate reduces stress, physical and mental fatigue in healthy people: A randomised, double-blind, placebo-controlled trial. Nutrients 6 : 2348-2359, 2014

27. Milesi MA, Lacan D, Brosse H, Desor D and Notin C: Effect of an oral supplementation with a proprietary melon juice concentrate (Extramel) on stress and fatigue in healthy people: A pilot, double-blind, placebo-controlled clinical trial. Nutr J 8: 40, 2009.

28. Camargo CA, Ganmaa D, Frazier AL, Kirchberg FF, Stuart JJ, Kleinman K, Sumberzul N and Rich-Edwards JW: Randomized trial of vitamin D supplementation and risk of acute respiratory infection in Mongolia. Pediatrics 130: e561-e567, 2012.

29. Zittermann A, Pilz S, Hoffmann H and März W: Vitamin D and airway infections: A European perspective. Eur J Med Res 21: 14, 2016.

30. Prasad AS, Beck FW, Bao B, Snell D and Fitzgerald JT: Duration and severity of symptoms and levels of plasma Interleukin-1 receptor antagonist, soluble Tumor Necrosis Factor Receptor, and adhesion molecules in patients with common coldtreated with zinc acetate. J Infect Dis 197: 795-802, 2008.

31. Prasad AS, Fitzgerald JT, Bao B, Beck FW and Chandrasekar PH: Duration of symptoms and plasma cytokine levels in patients with the common cold treated with zinc acetate. A randomized, double-blind, placebo-controlled trial. Ann Intern Med 133: 245-252, 2000
32. Choi SK, Baek SH and Choi SW: The effects of endurance training and thiamine supplementation on anti-fatigue during exercise. J Exerc Nutr Biochem 17: 189-198, 2013.

33. Suzuki M and Itokawa Y: Effects of thiamine supplementation on exercise-induced fatigue. Metab Brain Dis 11: 95-106, 1996.

34. Duthie SJ, Horgan G, De Roos B, Rucklidge G, Reid M, Duncan G, Pirie L, Basten GP and Powers HJ: Blood folate status and expression of proteins involved in immune function, inflammation, and coagulation: Biochemical and proteomic changes in the plasma of humans in response to long-term synthetic folic acid supplementation. J Proteome Res 9: 1941-1950, 2010.

35. World Medical Association: Declaration of Helsinki. Ethical principles for medical research involving human subjects. https://www. wma.net/policies-post/wma-declaration-of-helsinki-ethical-princi ples-for-medical-research-involving-human-subjects/, 2008.

36. ICH: Guideline for Good Clinical Practice E6 (R2), 2016.

37. Barrett B, Brown RL, Mundt MP, Thomas GR, Barlow SK, Highstrom AD and Bahrainian M: Validation of a short form Wisconsin upper respiratory symptom survey (WURSS-21). Health Qual Life Outcomes 7: 76, 2009.

38. Jaeschke R, Singer J and Guyatt GH: Measurement of health status. Ascertaining the minimal clinically important difference. Control Clin Trials 10: 407-415, 1989.

39. Hooper SL and Mackinnon LT: Monitoring overtraining in athletes. Recommendations. Sport Med 20: 321-327, 1995.

40. Fliege H, Rose M, Arck P, Walter OB, Kocalevent RD, Weber C and Klapp BF: The Perceived Stress Questionnaire (PSQ) reconsidered: Validation and reference values from different clinical and healthy adult samples. Psychosom Med 67: 78-88, 2005.

41. Ware J jr, Kosinski M and Keller SD: A 12-Item Short Health Survey: Construction of scales and preliminary tests of reliability and validity. Med Care 34: 220-233, 1996.

42. International Conference on Harmonization: ICH Topic E 9: Statistical principles for clinical trials, 1998.

43. Barrett B, Brown R, Voland R, Maberry R and Turner R: Relations among questionnaire and laboratory measures of rhinovirus infection. Eur Respir J 28: 358-363, 2006.

44. Nieman DC and Wentz LM: The compelling link between physical activity and the body's defense system. J Sport Heal Sci 8: 201-217, 2019.

45. Smyth AR, Cifelli PM, Ortori CA, Righetti K, Lewis S, Erskine P, Holland ED, Givskov M, Williams P, Cámara M, et al: Garlic as an inhibitor of Pseudomonas aeruginosa quorum sensing in cystic fibrosis-a pilot randomized controlled trial. Pediatr Pulmonol 45: 356-362, 2010.

46. Barrett B, Brown R, Rakel D, Rabago D, Marchand L, Scheder J, Mundt M, Thomas G and Barlow S: Placebo Effects and the Common Cold : A randomized controlled trial. Ann Fam Med 9: 312-322, 2011.

47. Obasi CN, Brown R, Ewers T, Barlow S, Gassman M, Zgierska A, Coe CL and Barrett B: Advantage of meditation over exercise in reducing cold and flu illness is related to improved function and quality of life. Influenza Other Respi Viruses 7: 938-944, 2013.

48. Spasov AA, Ostrovskij OV, Chernikov MV and Wikman G: Comparative controlled study of Andrographis paniculata fixed combination, Kan Jang and an Echinacea preparation as adjuvant, in the treatment of uncomplicated respiratory disease in children. Phyther Res 18: 47-53, 2004.

49. Cox AJ, Pyne DB, Saunders PU, Callister R and Gleeson M: Cytokine responses to treadmill running in healthy and illness-prone athletes. Med Sci Sports Exerc 39: 1918-1926, 2007.

50. Lawson LD and Gardner CD: Compositionstability, and bioavailability of garlicproductsbeing used in a clinical trial. J Agric Food Chem 53: 6254-6261, 2005.

51. Simpson R, Campbell J, Gleeson M, Krüger K, Nieman DC, Pyne DB, Turner JE and Walsh NP: Can exercise affect immune function to increase susceptibility to infection? Exerc Immunol Rev 26: 8-22, 2020.

52. Su QS, Tian Y, Zhang JG and Zhang H: Effects of allicin supplementation on plasma markers of exercise-induced muscle damage, IL-6 and antioxidant capacity. Eur J Appl Physiol 103: 275-283, 2008.

53. Niemelä M, Kangastupa $P$, Niemelä $\mathrm{O}$, Bloigu R and Juvonen $\mathrm{T}$ : Acute changes in inflammatory biomarker levels in recreational runners participating in a marathon or half-marathon. Sport Med Open 2: 21, 2016.

This work is licensed under a Creative Commons Attribution-NonCommercial-NoDerivatives 4.0 International (CC BY-NC-ND 4.0) License. 